\title{
Polymorphisms of GSTs in Lung Adenocarcinoma Patients Followed in the Context of a Biobank
}

\author{
Fotis Vlastos ${ }^{1}$, Georgios Hillas ${ }^{1}$, Nektarios Anagnostopoulos ${ }^{1}$, Jean Michel Vignaud ${ }^{2}$, Nadine Martinet ${ }^{3}$, \\ Nikolaos G. Koulouris ${ }^{1}$
}

${ }^{1}$ Department of Respiratory Medicine, University of Athens Medical School, "Sotiria” Hospital, Athens, Greece; ${ }^{2}$ The Centre for Biological Resources of Nancy/U724 INSERM, Central Hospital, Cour d'Anatomie, Nancy, France; ${ }^{3}$ Laboratory of Chemistry of Bioactive Molecules, UMR CNRS 6001, Nice, France.

Email: aris.anag@yahoo.gr

Received June $25^{\text {th }}, 2013$; revised July $28^{\text {th }}, 2013$; accepted August $6^{\text {th }}, 2013$

Copyright (C) 2013 Fotis Vlastos et al. This is an open access article distributed under the Creative Commons Attribution License, which permits unrestricted use, distribution, and reproduction in any medium, provided the original work is properly cited.

\begin{abstract}
Background: Lung Adenocarcinoma (ADC) has been recently associated with distinct molecular changes, leading to the development of molecular-based targeted therapy. The Nancy's Centre of Biological Resources ("Centre des Ressources Biologiques", CRB) is an ISO 9001-2000 certified biobank with biological material and follow-up data from lung cancer patients, which collected during the last 20 years. Objective: To estimate and compare the frequency of Glutathionne $S$-Transferase (GST) polymorphisms in a French population of ADC patients. Methods: A retrospective study was conducted by the CRB between 1988 and 2007: 296 consecutive patients operated upon for ADC and 447 healthy subjects were evaluated. Genomic DNA was obtained from peripheral blood samples collected in EDTA tubes. The DNA was extracted using proteinase $\mathrm{K}$ digestion and phenol: chloroform purification. The GST polymorphisms were studied with duplex SYBR Green q PCR using specific primers and results being read on melt curves. Results: Two GST classes were monitored during this research. The Mu class GST (GSTM) and the Theta class GST (GSTT) members. We studied the incidence of each genotype, as well as the GSTMT (combined Mu and Theta class) and null genotype in ADC and control patients. ADC patients had a higher incidence of the GSTM polymorphism ( $\mathrm{p}<0.0001$, $95 \%$ CI 1.63 - 3.24) and a lower incidence of the GSTT polymorphism ( $\mathrm{p}<0.0001,95 \% \mathrm{CI} 0.31-0.66)$ comparing to control. The null and GSTMT genotype had no significant statistical differences between the two groups. Conclusion: ADC patients were found to have a higher incidence of the GSTM genotype and a lower incidence of the GSTT genotype, compared to controls. Future studies may help elucidate the possible contribution of these genotypic differences in lung adenocarcinoma carcinogenesis or regarding the response to chemotherapy.
\end{abstract}

Keywords: GSTs; Molecular Signature; Lung Adenocarcinoma; Lung Cancer Risk

\section{Introduction}

Lung cancer was the first cause of cancer-related deaths in 2012 in the European Union with 353,000 deaths [1]. Tobacco smoking is the major attributable risk factor for the increasing prevalence of lung cancer across the world [2]. However, not all smokers are equally susceptible to tobacco-related carcinogens [3]. Thus, the identification of genes responsible for lung carcinogenesis susceptibility may allow researchers to perform screening programs and chemoprevention trials in subgroups of chronic smokers.

Lung Adenocarcinoma (ADC) has replaced squamous cell carcinoma as the most frequent histological subtype of lung cancer [4-7]. The Nancy's Centre of Biological
Resources (“Centre des Ressources Biologiques", CRB) is an ISO 9001-2000 certified biobank with biological material and follow-up data from lung cancer patients, which collected during the last 20 years. Nancy's CRB has been developed in the Nancy Central Hospital, aiming to recruit lung cancer patients willing to donate their samples for cancer research [8].

The French National Institute of Medical Research (INSERM) carried out this study aiming at the identification of biological tests associated with lung cancer risk. The tests included single nucleotide polymorphisms (SNP) of a family of detoxifying enzymes called Glutathione-S Transferases (GSTs). The activity of GSTs is dependent upon a steady supply of glutathione (GSH). 
The primary role of GSTs is to detoxify xenobiotics by catalyzing the nucleophilic attack by GSH on xenobiotic substrates, thereby preventing their interaction with crucial cellular proteins and nucleic acids $[9,10]$.

The aim of this study was to estimate and compare the frequency of GSTs polymorphisms in a French population of ADC patients. Therefore, we analysed genomic DNA from peripheral blood samples of lung adenocarcinoma patients and healthy controls.

\section{Materials and Methods}

\subsection{Patients}

A retrospective study was conducted by the CRB between 1988 and 2007: 743 subjects were evaluated (296 consecutive patients operated upon for ADC and 447 healthy subjects who were used as the control group).

The diagnosis of ADC was established by bronchial biopsies, cytological examination of sputum, bronchial washings and brushings and/or transthoracic fine needle aspiration of the lung lesion. All histological diagnoses had been confirmed by the same pathologists' panel. The histological classification was based upon the 2004 World Health Organization guidelines [11]. The sixth edition of the TNM (tumor, node, and metastasis) classification was used [12].

The CRB collection was initiated in 1988 to store tissue samples of patients operated for lung tumors as a resource for research purposes. From 2002, all survivors - new patients and control subjects - were invited to give their written informed consent, offer access to their medical records, and to donate biological material for research. Standard operating procedures were followed for all steps of sample collection and storage and for the retrieval of clinical annotations culminating in ISO 9001 certification of the biobank.

All the procedures were carried out according to the principles of the institutional guidelines and the study was approved by the local Ethics Committee.

\subsection{Methods}

Genomic DNA was obtained from peripheral blood samples $(12 \mathrm{ml})$ collected in EDTA tubes. The DNA was extracted using proteinase $\mathrm{K}$ digestion and phenol: chlo- roform purification. The Mu class GST (GSTM) and the Theta class GST (GSTT) SNPs were studied with duplex SYBR Green q PCR using the primers described in Table 1 and results being read on melt curves.

\subsection{Statistical Analysis}

The Hardy-Weinberg equilibrium was tested with the $\chi^{2}$ statistic for the goodness-to-fit (one degree of freedom). Statistical differences between groups were calculated by the $\chi^{2}$ or Fischer's exact test. Conditional analysis was used to obtain race, age and gender-adjusted crude odds ratios (OR). A p-value of $<0.05$ was considered significant. All analyses were performed using the statistical package SPSS version 17.0.

\section{Results}

Table 2 summarizes the main epidemiological and clinical characteristics of the study population. ADC patients were found to present a higher incidence of GSTM ( $p<$ $0.0001,95 \%$ CI $1.63-3.24)$ and a lower incidence of GSTT ( $p<0.0001,95 \%$ CI 0.31 - 0.66) genotypes comparing to control. The null and GSTMT genotype had no significant statistical differences between the two groups (Table 3).

\section{Discussion}

In our study, the GSTM genotype was more frequent among the ADC population (32.77\%) compared to the control group $(17.44 \%)(p<0.001)$, whereas the GSTT was less frequent $(15.87 \%$ compared to 29.30 respectively, $\mathrm{p}<0.001$ ).

The incidence and proportion of ADC cases has been steadily increasing for several decades worldwide, whereas the clinical course of the disease seems to be evolving, characterized by early development of distal metastases. Recent research in genetic epidemiology has improved our understanding of the lung carcinogenesis mechanisms and the multiple interactions between exposure and metabolism to known lung carcinogens, such as smoking products. This research allowed the identification of genomic polymorphisms involved in carcinogen metabolism. Polymorphisms of the genes encoding phase

Table 1. Primers used for the detection of the selected SNPs.

\begin{tabular}{|c|c|c|}
\hline \multirow{2}{*}{ Primers used for the detection of selected SNPs } & \multicolumn{2}{|c|}{ Reference } \\
\hline & Sens & Antisens \\
\hline GSTM & $\begin{array}{l}\text { CTG CCC TAC TTG ATT GAT GGG } \\
(\text { Chrl:110001735-110001755) }\end{array}$ & $\begin{array}{c}\text { CTG GAT TGT AGC AGA TCA TGC } \\
(\text { Chr1:110033445-110033465) }\end{array}$ \\
\hline
\end{tabular}


Table 2. Description of the cohort.

\begin{tabular}{|c|c|c|c|}
\hline & $\mathrm{ADC}$ & Control subjects & Total \\
\hline Total & 296 & 447 & 743 \\
\hline Males & 246 & 368 & 614 \\
\hline$\%$ & $83 \%$ & $82 \%$ & $85.3 \%$ \\
\hline Females & 50 & 79 & 129 \\
\hline$\%$ & $17 \%$ & $18 \%$ & $14.7 \%$ \\
\hline Age (years) & $60 \pm 9$ & $52 \pm 15$ & \\
\hline Tobacco (pack/years) & $39 \pm 24$ & $28 \pm 20$ & \\
\hline Never smokers & 28 & 50 & 107 \\
\hline$\%$ & $9 \%$ & $11 \%$ & $8.6 \%$ \\
\hline Survival (months) & $53 \pm 27$ & NA & \\
\hline$\%$ of the total & $23.8 \%$ & $36 \%$ & $100 \%$ \\
\hline
\end{tabular}

Table 3. The GSTs polymorphisms in patients with ADC. Comparison with controls.

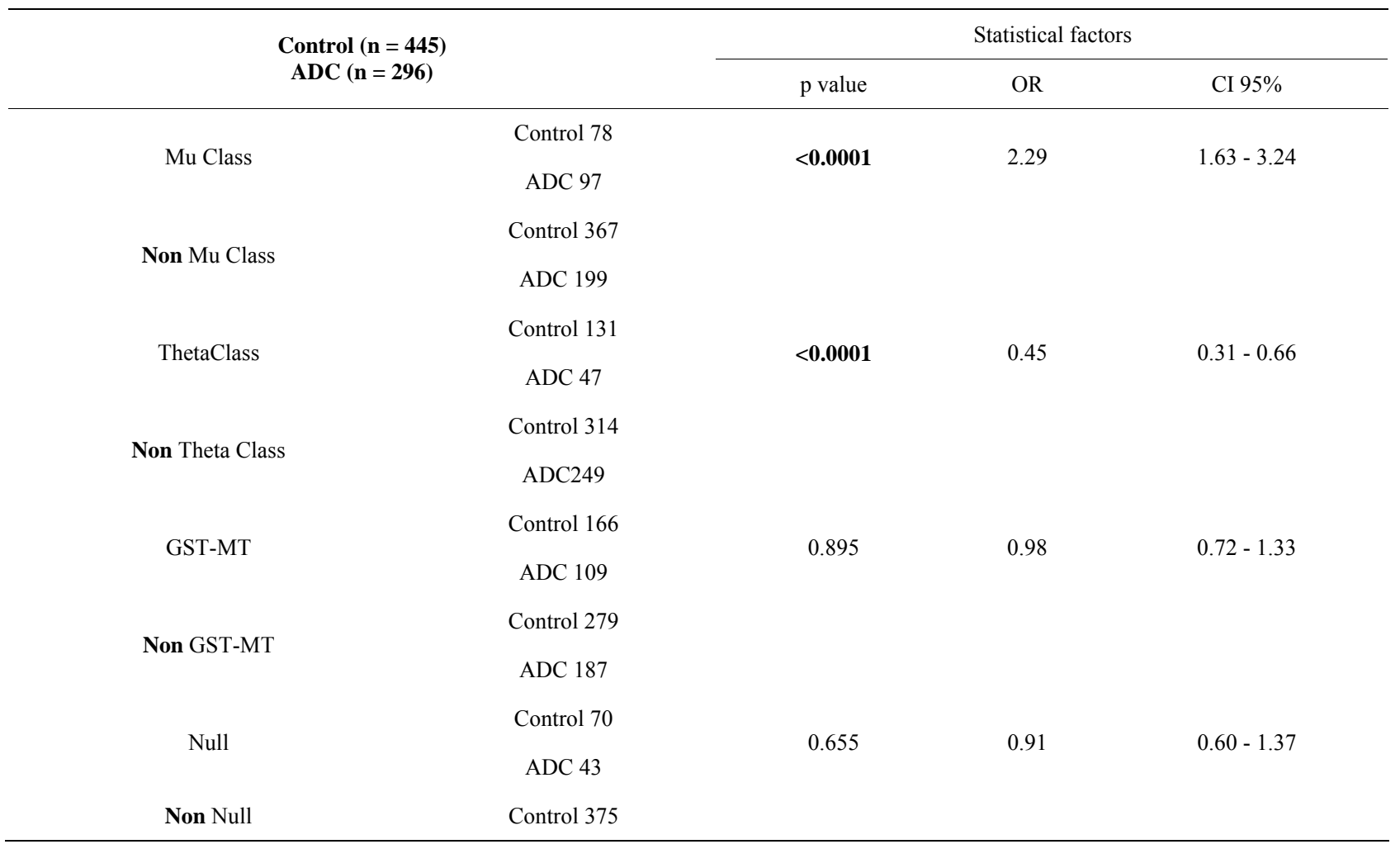

I and phase II xenobiotic metabolizing enzymes have been shown to be associated with susceptibility to lung cancer in a number of epidemiologic studies [13].

The functional polymorphisms in Glutathione STransferases (GSTs) genes may play an important role in ADC susceptibility. The GSTs form a family of enzymes catalyzing the detoxification of a wide range of eletrophilic substrates. They also play a significant role in phase II biotransformation of xenobiotics. Some genetic variants in the glutathione S-transferase genes, such as the GSTM1 null polymorphism, are known to abolish the related enzymatic activities. Individuals with the GSTM1 null genotype have been reported to have higher levels of polycyclic aromatic hydrocarbon-dGMP adducts in lung tissues comparing to those with the GSTM1 genotype [14]. Therefore, such genetic variants have been exten- 
sively studied as candidates for lung cancer susceptibility molecular screening.

We showed that ADC patients have a higher incidence of the GSTM genotype, being susceptible to SNPs in the $\mathrm{Mu}$ class of GSTs (usually resulting to the GSTM1 null genotype).

The data concerning the relation between GSTs polymorphisms and lung adenocarcinoma is conflicting so far. Whereas some studies show a weak positive correlation between GSTM1 and/or GSTT1 null genotypes and lung cancer risk, others show a negative association [15-19].

In particular, Zheng Ye and colleagues undertook a large meta-analysis of 130 published studies that had examined associations between one or several of five GST alleles and lung cancer [17]. A total number of 23.452 lung cancer cases and 30.397 controls were included. The two "null" polymorphisms in GSTM1 and GSTT1 showed either a very weak association with higher lung cancer risk or none at all.

Our findings are in harmony with the so far scientific data trends. In particular, the GSTM1 null polymorphism was associated weakly with a higher risk of ADC, whereas for the GSTT1 null polymorphism no correlation was found. This study may offer a paradigm of how biobanks may enable genome-based research in lung cancer field [20].

While pathology remains the cornerstone for diagnosis and the therapeutic decision, genomic-based approaches may enrich our insight into lung carcinogenesis and help not only diagnosis but also the selection of subjects at risk for chemoprevention trials [21,22].

\section{Conclusions}

The frequency of specific GSTs genotypes was related to higher risk of development of ADC. Further studies are needed to elucidate whether these techniques may serve as screening tools to assess ADC risk in smokers.

Future studies may help to elucidate the possible contribution of these genotypic differences in lung adenocarcinoma carcinogenesis or regarding the response to chemotherapy.

\section{REFERENCES}

[1] J. Ferlay, E. Steliarova-Foucher, J. Lortet-Tieulent, S. Rosso, J. W. Coebergh, H. Comber, D. Forman and F. Bray, "Cancer Incidence and Mortality patterns in Europe: Estimates for 40 Countries in 2012," European Journal of Cancer, Vol. 49, No. 6, 2013, pp. 1374-1403. doi:10.1016/j.ejca.2012.12.027

[2] B. W. Stewart and P. Kleihues, "World Cancer Report," IARC Press, Lyon, 2003.

[3] M. R. Spitz, Q. Wei, Q. Dong, et al., "Genetic Susceptibility to Lung Cancer: The Role of DNA Damage and Repair," Cancer Epidemiology, Biomarkers \& Prevention,
Vol. 12, No. 8, 2003, pp. 689-698.

[4] International Association for the Study of Lung Cancer/ American Thoracic Society/European Respiratory Society, "International Multidisciplinary Classification of Lung Adenocarcinoma," Journal of Thoracic Oncology, Vol. 6, No. 2, 2011, pp. 244-285.

doi:10.1097/JTO.0b013e318206a221

[5] M. P. Curado, B. Edwards, H. R. Shin, et al., "Cancer Incidence in Five Continents," Vol. IX, IARC Scientific Publications, Lyon, 2007.

[6] W. D. Travis, E. Brambilla, H. K. Muller-Hermelink, et al., "Pathology and Genetics. Tumours of the Lung, Pleura, Thymus and Heart," IARC Press, Lyon, 2004.

[7] M. J. Thun, C. A. Lally, J. T. Flannery, et al., "Cigarette Smoking and Changes in the Histopathology of Lung Cancer," Journal of the National Cancer Institute, Vol. 89, No. 21, 1997, pp. 1580-1586.

doi:10.1093/jnci/89.21.1580

[8] A. Charloux, E. Quoix, N. Wolkove, et al., "The Increasing Incidence of Lung Adenocarcinoma: Reality or Artifact? A Review of the Epidemiology of Lung Adenocarcinoma," International Journal of Epidemiology, Vol. 26, No. 1, 1997, pp. 14-23. doi:10.1093/ije/26.1.14

[9] F. Claudot, N. Malservet, C. Gallina-Muller, et al., "Le Centre de Ressources Biologiques Oncogenèse Thoracique," Bull Cancer, Vol. 91, 2004, pp. 201-203.

[10] P. D. Josephy, "Genetic Variations in Human Glutathione Transferase Enzymes: Significance for Pharmacology and Toxicology," Hum Genomics Proteomics, Vol. 2010, 2010, Article ID: 876940.

[11] J. D. Hayes, J. U. Flanagan and I. R. Jowsey, "Glutathione Transferases," Annual Review of Pharmacology and Toxicology, Vol. 45, 2005, pp. 51-88. doi:10.1146/annurev.pharmtox.45.120403.095857

[12] E. Brambilla, W. D. Travis, T. V. Colby, et al., "The New World Health Organization Classification of Lung Tumours," European Respiratory Journal, Vol. 18, No. 6, 2001, pp. 1059-1068. doi:10.1183/09031936.01.00275301

[13] L. H. Sobin and C. Wittekind, "International Union against Cancer (UICC), TNM Classification of Malignant Tumors," 6th Edition, Wiley-Liss, New York, 2002, pp. 99-103.

[14] L. C. Strong and C. I. Amos, "Inherited Susceptibility," In: D. Schottenfeld, J. G. Searle, J. F. Fraumeni Jr., Eds., Cancer Epidemiology and Prevention, Oxford University Press, New York, 1996, pp. 559-582.

[15] S. Kato, E. D. Browman, A. M. Harrigan, et al., "Human Lung Carcinogen-DNA Adduct Levels Mediated by Genetic Polymorphism in Vivo," Journal of the National Cancer Institute, Vol. 87, No. 12, 1995, pp. 902-907. doi:10.1093/jnci/87.12.902

[16] J. W. Wang, Y. F. Deng, et al., "Association of GSTM1, CYP1A1 and CYP2E1 Genetic Polymorphisms with Susceptibility to Lung Adenocarcinoma: A Case-Control Study in Chinese Population," Cancer Science, Vol. 94, No. 5, 2003, pp. 448-452. doi:10.1111/j.1349-7006.2003.tb01463.x 
[17] J. Wang, Y. Deng, J. Cheng, et al., "GST Genetic Polymorphisms and Lung Adenocarcinoma Susceptibility in a Chinese Population," Cancer Letters, Vol. 201, No. 2, 2003, pp. 185-193. doi:10.1016/S0304-3835(03)00480-4

[18] Z. Ye, H. Song, J. P. Higgins, et al., "Five Glutathione S-Transferase Gene Variants in 23,452 Cases of Lung Cancer and 30,397 Controls: Meta-Analysis of 130 Studies," PLoS Medicine, Vol. 3, No. 4, 2006, p. 91. doi:10.1371/journal.pmed.0030091

[19] N. Sunaga, T. Kohno, et al., "Contribution of the NQO1 and GSTT1 Polymorphisms to Lung Adenocarcinoma Susceptibility," Cancer Epidemiology, Biomarkers \& Prevention, Vol. 11, 2002, pp. 730-738.
[20] J. Altinisik, Z. B. Balta, G. Aydin, et al., "Investigation of Glutathione S-Transferase M1 and T1 Deletions in Lung Cancer," Molecular Biology Reports, Vol. 37, 2010, pp. 263-267. doi:10.1007/s11033-009-9673-5

[21] C. Lengauer, K. W. Kinzler and B. Vogelstein, "Genetic Instabilities in Human Cancers," Nature, Vol. 396, 1998, pp. 643-649. doi:10.1038/25292

[22] S. April and S. Ravi, "Biomarkers in Lung Cancer: From Early Detection to Novel Therapeutics and Decision Making," Biomarkers in Medicine, Vol. 2, No. 6, 2008, pp. 577-586. doi:10.2217/17520363.2.6.577 